\title{
Sleep Competing Activities and Sleep Problems in Minority College Students
}

\author{
Timothy Billings $\cdot$ Linda Berg-Cross
}

Received: 14 December 2013 /Revised: 13 July 2014 / Accepted: 23 July 2014 /Published online: 15 August 2014

(C) W. Montague Cobb-NMA Health Institute 2014

\begin{abstract}
This study addressed health disparities in sleep duration, sleep quality, and daytime sleepiness among African-American college students. To investigate evening behaviors promoting insufficient and inadequate sleep, we assessed electronic (e.g., computer and music) and nonelectronic (e.g., socializing) sleep competing activities (SCA). Students $(N=154)$ were recruited from a Historically Black College (HBCU). The results showed more dysfunction than has previously been reported in college populations, with HBCU students reporting very short sleep durations, high levels of daytime sleepiness, and poor sleep quality. These students engaged in many activities during the evening, and these activities predicted unhealthy sleep. Whereas electronic and nonelectronic activities significantly impacted sleep quality and sleep duration, only electronic activities were significantly related to sleep latency and only nonelectronic activities were significantly related to daytime sleepiness. We conclude that sleep is a major concern for college students and, in particular, racial minority college students. Understanding the role of SCA can help address the various environmental barriers for successful treatment of these young adults.
\end{abstract}

Keywords College students $\cdot$ Sleep quality $\cdot$ Excessive daytime sleepiness $\cdot$ Sleep competing activities

Previous research has highlighted the relationship between sleep problems and a variety of physical (e.g., diabetes mellitus, hypertension, obesity, and pain) [1-4] and mental health concerns [5]. The link between sleep problems and health risks continues throughout adulthood [2]. Evidence suggest that many college students report unhealthy sleep

T. Billings $(\bowtie) \cdot$ L. Berg-Cross

Howard University, Washington, DC, USA

e-mail: tim.billings.sleep@gmail.com parameters [6] and that younger adults report unhealthier sleep than older adults [7]. With an eye toward reducing health risks, this study focused on understanding how sleep competing activities (SCA) were related to sleep problems in a vulnerable minority college-age population. Given the large amount of time that students spend with electronics and its impact on sleep [8], coupled with their risk for circadian and delayed sleep phase problems [9], this study also investigated if activities based around electronics (e.g., computers and phone applications) affect sleep differently than nonelectronic sleep activities such as socializing or studying.

While there are many types of sleep problems, inadequate sleep duration, poor sleep quality, and excessive daytime sleepiness (EDS) are among the most common and most widely studied. Inadequate sleep duration and poor sleep quality often co-occur and produce EDS, although each of these three sleep problems can occur independently of one another. Achieving optimal healthy sleep requires adequate sleep duration, infrequent sleep onset and maintenance difficulties, and optimal sleep timing, all of which cumulatively produce an appropriate level of daytime alertness.

In college students, many of these previously mentioned sleep parameters are inadequate. For example, research over the past three decades suggests that the median number of hours slept by college students has decreased by more than $1 \mathrm{~h} /$ night [10] and that a majority of college students (70\%) report sleeping less than $6.5 \mathrm{~h} /$ night [11]. In the sparse research addressing health disparities, differences in total sleep time (TST) between African Americans and White college students have not been found [11]. Despite the nonsignificant differences found in college students' TST, research from adult samples suggests that differences exist and that African Americans report shorter TST than White Americans [12]. While group differences in sleep may be due, in part, to cultural differences that affect reporting, demand characteristics, or response to other situational factors, the consistency of 
such findings across many different types of data collection methods (e.g., computer administered, paper and pencil, inperson interviews, and sleep diaries) and settings (colleges, clinics, hospitals, and research centers) reduces the likelihood that these factors are the primary cause of the reported disparities [12].

Research investigating rates of EDS, independent of sleep duration, indicates the prevalence of daytime sleepiness is alarming. While there is significant variability among studies, prevalence rates range from $24 \%$ [6] to as high as $42 \%$ [13]. There is some evidence to suggest that African American students report greater daytime sleepiness than White students [13], although EDS in college students has only been minimally studied.

Unfortunately, restricted sleep and daytime sleepiness explains only part of the problem. Other parameters such as sleep latency and sleep maintenance often play a critical role in obtaining adequate sleep duration [14] and contribute to health-related quality of life [15]. In college students, long sleep latencies and fragmented sleep are common. In one sample of 313 college students, $33 \%$ took longer than $30 \mathrm{~min}$ to fall asleep, whereas other research has found that $43 \%$ of college students reported waking more than one time each night and $21 \%$ reported waking two to three times per night [16]. Similar sleep onset and maintenance difficulties have been reported between African American and White students [11]. These long sleep latencies and numerous nighttime awakenings profoundly affect sleep efficiency, sleep duration, sleep quality, and daytime sleepiness.

Evidence also suggests that maintaining a consistent sleep schedule is important to obtaining restorative, high-quality sleep [17], a construct known as "social jetlag." Yet, during college, this weekday-to-weekend bedtime variation is greatest. Lund and her colleagues [6] reported that $20 \%$ of respondents endorsed staying up all night at least one time in the last month, whereas $35 \%$ reported staying up until 3:00 a.m. at least one time per week [6].

Given the short sleep duration, frequent sleep onset and maintenance difficulties, and inconsistent weekday-toweekend sleep schedules reported by students, it is not surprising that poor sleep quality is frequently reported among this population. For example, an online survey of $1,125 \mathrm{col}-$ lege students found that $60 \%$ of participants reported poor sleep quality [6]. In another online sample of 710 university students, $50 \%$ of sampled students reported poor sleep quality [18]. Still more, in a sample of 468 nondepressed college students, $70 \%$ reported poor sleep quality [19]. These high rates of poor sleep quality have also been found cross culturally. In a sample of Brazilian university students $(N=710)$, $61 \%$ reported poor sleep quality [20]. Racial differences in sleep quality have not been reported between African American and White students [11], but again there are few studies in this area.
These high rates of insufficient sleep, poor sleep quality, and daytime sleepiness are significant for the health and wellbeing of college students, since it has been demonstrated that students with sleep problems have lower GPAs, greater depression rates, reduced retention for new learning, and problems dealing with stress [21-24]. Other studies have found that students with sleep problems report greater impulsivity which increases risky sexual behaviors as well as excess and dangerous use of drugs and alcohol $[25,26]$. Thus, it is critical to understand, assess, and treat sleep problems as a major public health problem.

Among college students, sleep problems are usually due to some combination of behaviorally induced insufficient sleep (i.e., sleep that is compromised due to voluntary behaviors of the sleeper) [27], delayed sleep phase syndrome (i.e., a circadian sleep disorder that shifts sleep onset $2 \mathrm{~h}$ later than normal) [9], insomnia, and obstructive sleep apnea (i.e., cessation of breathing during sleep due to obstructed airway) [28]. Cognitive behavioral therapy for insomnia (CBT-I) is the first-line treatment for sleep problems and is built around two techniques: stimulus control (using the bed only for sleeping) and/ or sleep restriction (manipulating the time to bed and time to rise on a predetermined schedule) [29]. While light manipulation is used in conjunction with these interventions for delayed sleep phase syndrome, and continuous positive airway pressure (CPAP) or oral appliances are the dominant treatments for obstructive sleep apnea, there have been no diagnosis specific interventions for behaviorally induced insufficient sleep. Moreover, CBT-I is fraught with obstacles and resistance in college students, as many live in shared sleeping environments, have schedules that vary considerably from semester-to-semester, and participate in social and academic activities that occur during normal sleeping times [6]. Understanding how different types of activities compete with sleep in college students may prove imperative if we are to protect college students' sleep.

Research regarding factors that influence sleep in collegeage students has primarily focused on alcohol, drugs, and stimulant use, and the evidence strongly suggests that these substances negatively impact sleep quality and daytime alertness $[11,30]$. Other environmental (e.g., homework and technology use) and interactional factors (e.g., social obligations and organized sports) can also derail sleep, but these SCA have been primarily investigated in adolescent samples [31-33] and little is known about their influence in collegeage students.

Internet use is one SCA that has been examined in college students, and the available research suggests that evening Internet use negatively impacts sleep. For example, in one study $73 \%$ of Brazilian university students $(N=710)$ who used the Internet between 7:00 p.m. and 12:00 a.m. also reported poor sleep quality [20]. In terms of sleep onset, sleep maintenance and early morning awakenings, the more young 
adults used the Internet and cell phones between 7:00 p.m. and 12:00 a.m., the more likely they were to report symptoms of insomnia (i.e., long sleep onset latency and sleep maintenance difficulties) than those not using the Internet or their cell phones during that time period [34]. However, there are many more activities that compete with sleep, including socializing, other technological devices, intramural and extramural activities, and school work and employment responsibilities. Many of these activities have been investigated in children and adolescents, whereas empirical studies documenting the impact of these behaviors on sleep in college students is only now being investigated. Furthermore, racial disparities in sleep have been minimally investigated and even less is known regarding the impact of evening activities on these students' sleep.

In light of this limited information, there is a distinct need for research investigating evening activities that contribute to unhealthy sleep patterns in college students (SCA). With college students' busy schedules limiting the time allotted to sleep, it is important to document the connection between the frequency of engaging in arousing activities during optimal sleep time and the resulting insufficient sleep duration, poor sleep quality and daytime sleepiness. Therefore, the current study had two goals. The first goal was to provide descriptive data on sleep parameters (e.g., TST, sleep quality, daytime sleepiness, and variations in weekday-to-weekend bedtimes) in a sample of racial minority students attending a Historically Black College/University (HBCU). Second, we examined the role of SCA in predicting sleep duration, sleep quality, and EDS among this racial minority sample of students. Specifically, it was hypothesized that individuals identified with unhealthy sleep parameters (e.g., short sleep duration, poor sleep quality, and EDS) would report more involvement in SCA than those with more healthy sleep parameters. We also explored if the frequency and intensity of the SCA or the type of SCA (electronic or nonelectronic) was related to sleep duration, sleep quality, and daytime sleepiness.

\section{Methods}

Participants/Procedures Participants $(N=158)$ were recruited from Introduction to Psychology classes at a HBCU and earned extra credit for participating in the study. Participants were recruited from the Psychology Research Pool at the HBCU. The Psychology Research Pool included all students enrolled in Introduction to Psychology classes and students received class credits for agreeing to participate in a study. Enrollment was restricted to students 18 years old and older. We did not restrict participation based on gender, ethnicity/ race, major, grade-point average, or any other variables. Participation times were distributed throughout the week and throughout the morning and afternoon time slots. No student opted out of the research requirement the semester data was gathered. Thus, we can cautiously assume that the data is representative of all students taking the course. Students were given a brief explanation of the purpose of the study (examining interpersonal relations), and informed consent was obtained before students completed the questionnaires. The Institutional Review Board (IRB) approved the methods and procedures used in this study, and paper and pencil questionnaires were completed by students anonymously. The proportion of female students who participated in the study ( $75 \%$ ) mirrored the disproportionate rates found for students enrolled in Introduction to Psychology classes.

Measures The Pittsburgh Sleep Quality Index (PSQI) and the Epworth Sleepiness Scale (ESS) comprised the majority of the outcome variables for this study. Additionally, demographic information and questions pertaining to SCA were collected. The ESS is a self-report questionnaire derived from observations about the nature and occurrence of daytime sleepiness [35]. ESS scores greater than 10 are typically used to indicate high levels of daytime sleepiness [36]. Although the ESS has been used extensively with adults, studies have examined its functionality in adolescents and found it to be a valid and reliable measure of daytime sleepiness [37]. Scores may be artificially lowered because some of the ESS questions may not be particularly relevant to college students. The PSQI is a 19-item self-report questionnaire for evaluating sleep quality over a 1-month duration [38]. The questions are compiled into 7 component scores with scores ranging from 0 to 3 and a total score with a range between 0 and 21 . The questionnaire and the scoring instructions for calculating index scores can be obtained on-line (http://www.sleep.pitt.edu/default.asp). The psychometric properties of the PSQI have been thoroughly investigated [38, 39]. Typically, a cut-off score of 5 is used to distinguish individuals with normal sleep quality from those with poor sleep quality.

Sleep habits were assessed using a 28 -item questionnaire developed by the investigators. The questionnaire has two parts. The first section elicits information about sleep habits such as sleep onset and wake times, napping behavior, schoolday wake times, and nighttime awakenings (Sleep Habits Scale). The second section examines 12 different activities that compete with sleep (The SCA scale). To date, there has not been a survey investigating various types of activities that impact sleep. The SCA scale is unique in that it assesses a variety of activities that can compete with sleep, including television viewing, studying and homework, video games and Internet use, texting friends and socializing, eating, playing with a pet, taking care of family members, listening to music, doing chores, and playing sports. Items for the SCA scale were chosen by reviewing the adolescent sleep literature on this topic as well as discussions with parents and researchers in the field about activities that interfere with sleep. The 
ratings in the SCA scale were based on the likelihood and frequency with which students engaged in each activity once they were already sleepy and ready for bed. Hence, we were assessing activities that were actually competing with the need and desire to sleep. Summing the responses to the individual activities produces a total SCA score.

There is also a weighted SCA score that was derived by multiplying the number of days in a week engaging in a activity (frequency) by the length of time spent engaging in the activity (duration). The weighted SCA score was important because it allowed us to examine intensity/frequency of SCA as well as the cumulative number of different SCA, which is reflected in the total SCA score. So while someone may have a low SCA score, if they engage in those few behaviors every day, the weighted SCA score may be more helpful in assessing its impact.

Items on the SCA scale can also be combined in a way that allows for an evaluation of the impact of activities involving electronics (E-SCA score; i.e., television use, Internet use, texting, etc.) and those activities not involving electronic devices (NE-SCA score; i.e., socializing, exercising, etc.), both of which can be computed for frequency scores and weighted frequency of (activity) multiplied by duration of the (activity) scores. This allowed us to examine if duration of an activity magnified any frequency effects that we found. Thus, looking at electronic and nonelectronic scores separately was important because electronic devises may affect circadian rhythms in a unique way (E-SCA score) and nonelectronic activities (NE-SCA score) may have unique interpersonal effects that impact the relaxation response and sleep. For the SCA score, the range of possible scores is between 0 and 70 , and the weighted SCA score has a range of scores between 0 and 280. All analyses reported in this article are based on weighted SCA scores and only included 10 of the 12 items. Overall, Cronbach's alpha for the weighted SCA scale was $\alpha=0.652$, within the range expected for a scale in the early stages of development [40]. Co-efficient alpha for the electronics subscale was $\alpha=.647$ and for the nonelectronic activities subscale it was $\alpha=.400$.

\section{Analytical Methods}

Descriptive analyses were conducted to characterize the sample. The main sleep outcome variables (sleep quality, sleep duration, and EDS) were all continuous and normally distributed. Additionally, the weighted SCA total score was also continuous and normally distributed. First, analyses were conducted on the individual items of the SCA questionnaire. Independent $t$ tests (for normally distributed data) and MannWhitney-Wilcoxon tests (for not normally distributed data) were used to examine differences in sleep duration, sleep quality, and EDS [41]. Next, independent $t$ tests (for normally distributed data) and Mann-Whitney-Wilcoxon tests (for not normally distributed data) were used to examine differences between students with healthy versus unhealthy sleep parameters and their scores on the total SCA scale and the two subscales (electronic and nonelectronic SCA). Pearson correlation coefficients were used to assess the relationships between the SCA total score and its two subscales (electronic and nonelectronic subscales), and the three main sleep outcome measures (sleep quality, sleep duration, and EDS). Pearson correlation coefficients were also computed for other sleep variables including three composite scores from the PSQI (sleep duration composite, sleep disturbance composite score, and the sleep quality composite score) as well as school-day sleep duration, and past-month sleep duration. Analyses were conducted with SPSS version 17.0 (SPSS Inc., Chicago, IL).

\section{Results}

The participants' mean age was 19 years $(\mathrm{SD}=1.47)$. Seventyfive percent of the sample was female, $25 \%$ were male, and one person failed to identify their sex $(N=154)$. Due to the preponderance of female participants in this study, sex differences were not consistently analyzed; however, the disproportionately high number of female participants generally reflects the enrollment ratio for this HBCU and thus allows for generalizations about undergraduate students in this setting. A majority of students were African American (95\%,n=142). The other $5 \%$ of the sample were nonCaucasian minority students (e.g., Asian, etc).

\section{Sleep Problems}

Students slept on average $6 \mathrm{~h} /$ night $(\mathrm{SD}=1.19)$ and a majority of students $(62 \%)$ were identified as short sleepers (sleeping less than $6.5 \mathrm{~h} /$ night). Very few participants $(9 \%)$ reported sleeping $8 \mathrm{~h}$ or more per night. The average sleep onset latency was $22 \mathrm{~min}(\mathrm{SD}=28.30)$, but $21 \%$ of students took 30 min or more to fall asleep. Mean PSQI total score for the sample was $6.9(\mathrm{SD}=2.93)$, demonstrating that the average student experienced clinically significant poor sleep quality. Poor sleep quality (PSQI total scores greater $>5$ ) was reported by $63 \%$ of participants. The average ESS total score was 11.7 $(\mathrm{SD}=3.41)$, indicating that a majority of students experienced EDS. EDS (ESS scores $>10$ ) was reported by $61 \%$ of the sample.

The average school-day wake time was 7:50 a.m. $(\mathrm{SD}=$ $74 \mathrm{~min}$ ) with only $12 \%$ of students waking later than 9:00 in the morning. Average bedtime on school days was 12:57 a.m. $(\mathrm{SD}=73 \mathrm{~min}$ ) and on weekends was 2:49 a.m. ( $\mathrm{SD}=90 \mathrm{~min}$ ). Yet on weekends, $97 \%$ of students went to bed after 12:00 a.m. and $63 \%$ went to bed at 3:00 a.m. or later. The 
average weekday-to-weekend variation in bedtime was $2 \mathrm{~h}$ $(\mathrm{SD}=71 \mathrm{~min})$; yet, $27 \%$ of students had variations of $3 \mathrm{~h}$ or more. Napping was frequent in this sample of college students with $28 \%$ of participants never napping, $67 \%$ reported napping occasionally and $5 \%$ of participants reported daily napping.

\section{Sleep Competing Activities}

Of the $12 \mathrm{SCA}$, two were removed from the analysis because of the very low rates of positive responses to these items. These items were playing with pets (97\% reported zero), and taking care of siblings or family members $(90 \%$ reported zero). The most frequently reported SCA included texting friends, eating, Internet use, socializing, listening to music, and watching television. Note that the data reported refers to SCA that occur only after the student is sleepy and ready for bed and does not reflect total daily usage.

Texting was most common and occurred on average five times per week ( $\mathrm{SD}=2.35$ ); yet, $47 \%$ of participants reporting texting while sleepy every night. Students reported completing homework four $(\mathrm{SD}=1.83)$ nights per week, and Internet use occurred on average four nights per week $(\mathrm{SD}=2.21)$. Socializing during normal sleeping hours occurred three $(\mathrm{SD}=2.02)$ nights per week, whereas listening to music was reported on average three $(\mathrm{SD}=2.46)$ nights per week. Watching television during normal sleeping time occurred on average three $(\mathrm{SD}=2.35)$ nights per week. Eating late at night was also reported three nights per week $(\mathrm{SD}=1.99)$, whereas completing chores $(\mathrm{M}=1$ day/week $\mathrm{SD}=1.37)$, video game use $(\mathrm{M}=0.3, \mathrm{SD}=0.88)$, and playing sports $(\mathrm{M}=0.1$, $\mathrm{SD}=0.59)$ were infrequently reported as occurring after sleepiness had set in. Significant sex differences were found between two sleep competing behaviors. Female participants $(\mathrm{M}=4.48, \mathrm{SD}=1.80)$ reported more time spent doing homework than male participants $(\mathrm{M}=3.78, \mathrm{SD}=1.90 ; t(147)=-$ 2.033, $p=0.044)$. Male participants reported significantly more time spent playing video games $(\mathrm{M}=0.81, \mathrm{SD}=1.35)$ than female participants $(\mathrm{M}=0.12, \mathrm{SD}=.57)$ (video games: $t$ $(40.25)=3.04, p=0.004)$. No significant sex differences were found on the weighted SCA score.

Independent $t$ tests (for normally distributed data) and Mann-Whitney-Wilcoxon tests (for not normal distributed data) were used to determine whether individuals with short sleep duration (sleep duration $<6.5 \mathrm{~h} / \mathrm{night}$ ), poor sleep quality (PSQI total score $>5$ ), and EDS (ESS score $>10$ ) engaged more frequently in SCA than students with more healthy sleep parameters and the results confirm expectations. Students who slept less than $6.5 \mathrm{~h} /$ night scored higher on four weighted SCA (television use: $U=2,011.5, p=0.016$, late night eating: $U=$ $1,729.5, p=0.001$, socializing with friends: $t(143.6)=-2.342$, $p=0.021$, and music listening: $U=1,924.5, p=0.007)$. Individuals who reported poor sleep quality (PSQI score $>5$ ) scored higher on three of the weighted SCA (television use: $U=$ 2,079, $p=0.038$, Internet use: $t(147)=-1.984, p=0.049$, sports: $U=2,436.0, p=0.037$ ). Individuals with EDS (ESS score $>10$ ) scored higher on all of the weighted SCA items than those without EDS; yet, only sports involvement was statistically significant $(U=2,478, p=0.030)$.

These results suggest that the individual SCA impact a variety of sleep parameters. Individuals with poor sleep quality (PSQI score $>5$ ) scored higher than students with healthier sleep quality on five of the ten SCA, students with short sleep duration $(<6.5 \mathrm{~h} / \mathrm{night})$ scored higher than students with longer sleep duration on four out of ten SCA, and individuals with EDS (ESS score $>10$ ) scored higher than students with less daytime sleepiness on two SCA. Both short sleeping students $(<6.5 \mathrm{~h} /$ night $)$ and students with poor sleep quality (PSQI $>5$ ) scored significantly higher on nighttime television use and music listening, whereas poor quality sleepers also reported more Internet use, chores, and sports than students who reported healthier sleep quality. Chores and sports were also more frequent among individuals with EDS. Students with short sleep duration $(<6.5 \mathrm{~h} / \mathrm{night})$ also reported more late night eating and socializing than students with more healthy sleep durations.

Similar to the previous analyses, independent $t$ tests were used to determine if students with short sleep duration, poor sleep quality, and EDS reported higher scores on the scale assessing the cumulative impact of all SCA. Overall, the average weighted total SCA score (weighted SCA scale) was $65.8(\mathrm{SD}=31.68)$ with a range of scores from 9 to 171 . Results from these analyses indicated that only individuals reporting poor sleep quality (PSQI total score $>5$ ) and students sleeping less than $6.5 \mathrm{~h} /$ night reported significantly higher weighted SCA scores than students reporting healthier sleep parameters (see Table 1). These results indicated that students with short sleep duration and poor sleep quality spend a considerable amount of time engaging in SCA while sleepy and at the expense of their sleep.

Assessing electronic versus nonelectronic SCA, independent $t$ tests indicated that students with short sleep duration (less than $6.5 \mathrm{~h}$ sleep/night) scored higher than long sleepers on the weighted electronic SCA subscale (weighted E-SCA) and the weighted nonelectronic SCA subscale (weighted NESCA) (see Table 1). Similarly, individuals who reported poor sleep quality (PSQI score $>5$ ) also scored higher on the weighted electronic activities and nonelectronic activities subscales. Individuals reporting EDS (ESS score $>10$ ) reported more nonelectronic SCA, but none of these tests reached statistical significance. Thus, daytime sleepiness is less affected by electronic and nonelectronic SCA than the other two measures of sleep (sleep quality and sleep duration).

To evaluate hypothesis two, Pearson correlation coefficients were used to assess the relationship between the three scales assessing SCA and the sleep outcome measures (see 
Table $1 t$ tests for sleep competing activities scale

\begin{tabular}{|c|c|c|c|c|c|c|c|c|c|}
\hline $\begin{array}{l}\text { Scale/ } \\
\text { subscale }\end{array}$ & $\begin{array}{l}\text { Normal sleep } \\
\text { duration } \\
(N=57)\end{array}$ & $\begin{array}{l}\text { Short sleep } \\
\text { duration } \\
(N=93)\end{array}$ & $t$ score & $\begin{array}{l}\text { No EDS } \\
(N=59)\end{array}$ & $\begin{array}{l}\text { EDS } \\
(N=91)\end{array}$ & $t$ score & $\begin{array}{l}\text { Normal sleep } \\
\text { quality } \\
(N=56)\end{array}$ & $\begin{array}{l}\text { Poor sleep } \\
\text { quality } \\
(N=94)\end{array}$ & $t$ score \\
\hline $\begin{array}{l}\text { Weighted } \\
\text { SCA }\end{array}$ & $\begin{array}{l}\mathrm{M}=55.4 \\
\quad(\mathrm{SD}=30.21)\end{array}$ & $\begin{array}{l}\mathrm{M}=72.1 \\
\quad(\mathrm{SD}=31.02)\end{array}$ & $-3.229 * * *$ & $\begin{array}{l}\mathrm{M}=60.7 \\
\quad(\mathrm{SD}=30.41)\end{array}$ & $\begin{array}{l}M=69.1 \\
\quad(S D=32.20)\end{array}$ & ns & $\begin{array}{l}M=56.4 \\
\quad(S D=29.33)\end{array}$ & $\begin{array}{l}M=71.3 \\
\quad(S D=31.86)\end{array}$ & $-2.847 * *$ \\
\hline $\begin{array}{r}\text { Weighted } \\
\text { E-SCA }\end{array}$ & $\begin{array}{l}M=30.9 \\
\quad(S D=23.12)\end{array}$ & $\begin{array}{l}\mathrm{M}=40.2 \\
\quad(\mathrm{SD}=22.66)\end{array}$ & $-2.402 *$ & $\begin{array}{l}M=34.1 \\
\quad(S D=22.18)\end{array}$ & $\begin{array}{l}M=38.3 \\
\quad(S D=23.81)\end{array}$ & ns & $\begin{array}{l}M=30.9 \\
\quad(S D=20.69)\end{array}$ & $\begin{array}{l}\mathrm{M}=40.1 \\
\quad(\mathrm{SD}=24.03)\end{array}$ & $-2.375^{*}$ \\
\hline $\begin{array}{l}\text { Weighted } \\
\text { NE-SCA }\end{array}$ & $\begin{array}{l}\mathrm{M}=24.5 \\
\quad(\mathrm{SD}=13.36)\end{array}$ & $\begin{array}{l}\mathrm{M}=31.9 \\
\quad(\mathrm{SD}=15.37)\end{array}$ & $-3.030 * *$ & $\begin{array}{l}M=26.5 \\
\quad(S D=13.94)\end{array}$ & $\begin{array}{l}\mathrm{M}=30.8 \\
\quad(\mathrm{SD}=15.55)\end{array}$ & ns & $\begin{array}{l}M=25.5 \\
\quad(S D=13.40)\end{array}$ & $\begin{array}{l}\mathrm{M}=31.2 \\
\quad(\mathrm{SD}=15.61)\end{array}$ & $-2.284^{*}$ \\
\hline
\end{tabular}

$n s$ not significant, $M$ mean, $S D$ standard deviation, $S C A$ sleep competing activities scale, $E-S C A$ electronics sleep competing activities scale, $N E-S C A$ nonelectronic sleep competing activities scale, $E D S$ excessive daytime sleepiness

${ }^{*} p<0.05 ; * * p<0.01 ; * * * p<0.001$

Table 2). The weighted SCA scale was significantly correlated with three outcome parameters including: the PSQI Duration of Sleep composite score, average sleep duration during the past month, and average school-day sleep duration.

Both the electronic and nonelectronic subscales were highly correlated with many of the sleep disturbance measures (Table 2). The weighted E-SCA scale was significantly correlated with four outcome measures: school-day sleep duration, PSQI Duration of Sleep Index, PSQI Sleep Disturbance Index, and the PSQI Total Score. The weighted NE-SCA scale was significantly correlated with three sleep parameters including: EDS, PSQI Duration of Sleep Index, and PSQI Overall Sleep Quality Index. This confirms previous findings, again demonstrating the consistent relationship between SCA and sleep disturbances in this population.

Table 2 Sleep parameters and SCA scores: correlations

\begin{tabular}{llll}
\hline & \multicolumn{2}{l}{ Sleep competing activities subscales } \\
\cline { 2 - 4 } & $\begin{array}{l}\text { Weighted } \\
\text { SCA }\end{array}$ & $\begin{array}{l}\text { Weighted } \\
\text { E-SCA }\end{array}$ & $\begin{array}{c}\text { Weighted } \\
\text { NE-SCA }\end{array}$ \\
\hline PSQI (total) & $0.225^{* *}$ & $0.206^{*}$ & 0.155 \\
ESS (total) & 0.157 & 0.078 & $0.211^{* *}$ \\
PSQI (SlpDurat) & $0.299^{* *}$ & $0.217^{* *}$ & $0.294^{* *}$ \\
PSQI (SlpDisturb) & $0.216^{* *}$ & $0.255^{* *}$ & 0.060 \\
PSQI (SlpQual) & 0.157 & 0.098 & $0.180^{*}$ \\
School day (SlpDurat) & $-0.258^{* *}$ & $-0.190^{*}$ & $-0.250^{* *}$ \\
SlpDurat (past month) & $-0.289^{* *}$ & $-0.201^{*}$ & $-0.299^{* *}$ \\
\hline
\end{tabular}

PSQI (total) Pittsburgh Sleep Quality Index (global sleep quality), ESS (total) Epworth Sleepiness Scale Total Score, PSQI (SlpDurat) PSQI Sleep Duration Index Score, PSQI (SlpDisturb) PSQI (Sleep Disturbance Index), PSQI (SlpQual) PSQI (sleep quality), SlpDurat (past month) pastmonth sleep duration

${ }^{*} p<0.05 ;{ }^{* *} p<0.01$

\section{Discussion}

Sleep is a major concern among college students and this was confirmed by a majority of the analyses. The students from this sample reported short sleep duration and high levels of daytime sleepiness. Whereas Lund and colleagues [6] found that $29 \%$ of their sample slept $8 \mathrm{~h}$ or more per night, only $9 \%$ of participants from this sample reported sleeping $8 \mathrm{~h}$ or more per night. In addition, our findings indicated that $62 \%$ of students were sleeping less than $6.5 \mathrm{~h} / \mathrm{night}$, whereas Lund and colleagues [6] reported that only $25 \%$ of their sample were sleeping less than $6.5 \mathrm{~h} /$ night. In the Lund and colleagues [6] study, participants were predominantly white. Still more, Lund and her colleagues [6] reported a mean TST of $7.02 \mathrm{~h}$ $(\mathrm{SD}=1.15)$, and Medeiros and colleagues [42] reported a mean TST of $6.87 \mathrm{~h}(\mathrm{SD}=93 \mathrm{~min})$. Yet, results from our study indicated that the average TST was shorter, with students reporting an average sleep duration of $6 \mathrm{~h}(\mathrm{SD}=1.19)$ per night. These findings suggest that the minority college students in this sample experience considerably more sleep problems than others.

Other important findings from this study include those related to EDS and sleep quality. First, the levels of daytime sleepiness reported by this sample were much higher than those reported in previous studies that focused on predominantly White or mixed race samples. Lund and her colleagues [6] found that $25 \%$ of their sample reported EDS (ESS total $>10$ ), whereas $42 \%$ reported EDS in the study by Edens [13]. In this sample, $61 \%$ of participants reported EDS. These findings make sense, given that students in this sample reported shorter sleep durations than those reported in the other studies. In addition, it is common for individuals who restrict sleep to also report short sleep onset latencies and this was also reported in this sample. Whereas Forquer and colleagues [16] reported that $33 \%$ of their participants took $30 \mathrm{~min}$ or longer to fall asleep, only $21 \%$ of our sample took $30 \mathrm{~min}$ or more to fall asleep, probably reflecting the greater sleep debt in our 
sample. Second, the results found here concerning sleep quality were similar to results reported in other recent studies [6, 20]. Lund and colleagues [6] reported that $60 \%$ of sampled students reported poor sleep quality, and Mesquita and Reimao [20] reported that $61 \%$ of sampled students reported poor sleep quality. In this sample, we found that $63 \%$ were identified as poor quality sleepers which were similar to the rates of poor sleep quality reported in the previously mentioned studies.

Another important finding from this study has to do with the degree to which students were delaying weekend bedtimes. Consistent with previous studies, a pattern of delaying weekend bedtimes was found in the present sample of students. Yet, students in this sample were delaying weekend bedtimes by a little over an hour more than was reported in previous studies. Lund and colleagues [6] found mean weekday bedtime was 12:17 a.m. and weekend bedtime was 1:44 a.m., while in our sample the mean weekday bedtime was 12:57 a.m. (40 min later than reported by Lund) and weekend bedtime was 2:49 a.m. (65 min later than reported by Lund). In our sample, over a quarter of participants had weekday-to-weekend variations in sleep of $3 \mathrm{~h}$ or more, which is concerning given how inconsistent sleeping patterns impact circadian rhythm timing [43].

The second aim of this study was to examine the impact of SCA on minority college students' sleep. This research suggests that both activities including electronic devices (e.g., television and Internet use and listening to music) and activities such as socializing, homework, extracurricular sports, and chores impact sleep duration, sleep quality and daytime sleepiness. The six most frequently reported SCA in this sample of students included texting, eating, Internet use, socializing, listening to music, and watching television. Although occurring less frequently in this sample, chores and playing sports also significantly influenced sleep parameters.

Both E-SCA and NE-SCA were related to at least one sleep quality measure and both measures of sleep duration. While engaging in activities involving electronics (weighted E-SCA subscale) predicted overall sleep quality, nonelectronic activities (weighted NE-SCA subscale) predicted daytime sleepiness. This suggests that repeatedly compromising sleep by hyper-focusing on preferred electronic activities, night after night, affects both the ability to quickly go to sleep and sleep quality, whereas engaging in multiple nonelectronic SCA impacts sleep quality, later bedtimes and daytime sleepiness. One explanation for this finding is that television, computer use, and cell phones emit bright lights that delay the production of melatonin which is a biochemical marker used to signal sleep onset and also affects other aspects of sleep quality [43]. Yet, the fact that multi-hour consumption of specific NE-SCA also affected sleep quality suggests a general arousal factor associated with intense involvement (be it socializing, homework, or the Internet) that affects sleep quality. This is contrary to the healthy benefits normally associated with psychologically engrossing activities that produce "flow" [44], but is more consistent with research by Gradisar and colleagues [7] which found that, among activities involving electronic devices, those activities that were more interactive (e.g., cell phones, video games, and computers) were more likely to disrupt sleep. Future research needs to tease out why students might be engrossed in activity and not achieving beneficial flow, or whether despite the beneficial effects of flow, nighttime activities generally impair sleep quality.

Two other noteworthy results were found in this study. First, this study raises concerns about the relationship between listening to music and sleep. Listening to music at night is common among adolescents and college students and is often encouraged for people who have sleep-onset difficulties. Yet, the results here suggest a significant relationship between listening to music at night and shorter sleep durations and poorer sleep quality. Although we did not differentiate between listening to music alone in an environment conducive of sleep and music listening in other situations (i.e., social outings), these findings raise an important concern and warrant further investigation. Similarly, specific aspects of the music itself may moderate the relationship between music and healthy sleep. Therefore, future studies that survey the type of music will also be important.

Although the present research provides important insights into sleep in an understudied population, there were some limitations that warrant attention. For example, a standard introductory psychology sampling technique was used, where volunteers were given credit for participation. As with all research that uses convenience samples, the results found here cannot be generalized beyond this sample. Also, given the small sample size multivariate modeling was not used and future studies are required to provide reliability for the SCA scale. Despite these limitations, the results found here warrant future investigation. Regarding the high rates of EDS, short sleep duration, and large variations in weekday-to-weekend bedtimes, future studies should determine whether the unhealthy sleep parameters found here represent race disparities in sleep or whether these differences reflect other characteristics such as differences in campus-wide norms or advances in technology. Given the relatively small sample size and large percentage of female students, future research should seek to replicate the findings reported here and determine if females and males engage in these activities with the same frequency and duration. Also, it is important for future research to investigate how electronic and NE-SCA may differentially impact sleep, as there is evidence that sleep quality may be more impacted by E-SCA than NE-SCA.

In summary, sufficient sleep is achieved through biological and environmental pathways. Among college students 
the evidence suggests that healthy sleep parameters are deteriorating, and the results here confirm this notion and further suggest that HBCU college students are disproportionately compromising their sleep despite its negative health consequences. From these results, there is no doubt that SCA impact sleep. In addition, these findings also suggest the type of activity (i.e., electronic devices versus nonelectronic devices) influences how sleep is impaired. With the precipitous decline in healthy sleep in college students, it is imperative to understand the factors that contribute to inadequate and insufficient sleep as these environmental conditions often present as significant treatment barriers. Future research which seeks to understand any racial disparities in sleep parameters as well as any disparities regarding the impact of SCA is critical to combat this growing health concern. Given the plethora of research highlighting unhealthy sleep in college students, it will be important for college counseling centers to recognize the unhealthy practices that contribute to college students' sleep problems. Moreover, it will also be important for college administrators to develop initiatives that educate students about the health consequences of poor sleep and to conduct more research to better understand the various environmental factors that contribute to unhealthy sleep.

Acknowledgments All procedures followed were in accordance with the ethical standards of the responsible committee on human experimentation (institutional and national) and with the Helsinki Declaration of 1975 , as revised in 2000 (5). Informed consent was obtained from all patients for being included in the study. No animal or human studies were carried out by the authors for this article.

Conflict of Interest Timothy Billings and Linda Berg-Cross declare that they have no conflict of interest.

\section{References}

1. Ohayon MM. Pain sensitivity, depression, and sleep deprivation: links with serotoninergic dysfunction. J Psychiatr Res. 2009;43(16): $1243-5$.

2. Guo X, Zheng L, Wang J, Zhang X, Zhang X, Li J, et al. Epidemiological evidence for the link between sleep duration and high blood pressure: a systematic review and meta-analysis. Sleep Med. 2013;14(4):324-32. doi:10.1016/j.sleep.2012. 12.001.

3. Cappuccio FP, D'Elia L, Strazzullo P, Miller MA. Quantity and quality of sleep and incidence of type 2 diabetes: a systematic review and meta-analysis. Diabetes Care. 2010;33(2):414-20. doi:10.2337/ dc09-1124.

4. Morselli L, Leproult R, Balbo M, Spiegel K. Role of sleep duration in the regulation of glucose metabolism and appetite. Best Pract Res Clin Endocrinol Metab. 2010;24(5):687-702.

5. Brooks PR, Girgenti AA, Mills MJ. Sleep patterns and symptoms of depression in college students. Coll Stud J. 2009;43(2):464-72.
6. Lund HG, Reider BD, Whiting AB, Prichard JR. Sleep patterns and predictors of disturbed sleep in a large population of college students. J Adolesc Health. 2010;46(2):124-32.

7. Gradisar M, Wolfson AR, Harvey AG, Hale L, Rosenberg R, Czeisler CA. The sleep and technology use of Americans: findings from the National Sleep Foundation's 2011 Sleep in America Poll. J Clin Sleep Med. 2013;9(12):1291-9. doi:10. $5664 / j \operatorname{csm} .3272$.

8. Higuchi S, Motohashi Y, Liu Y, Maeda A. Effects of playing a computer game using a bright display on presleep physiological variables, sleep latency, slow wave sleep and REM sleep. J Sleep Res. 2005;14(3):267-73.

9. Brown FC, Soper B, Buboltz Jr WC. Prevalence of delayed sleep syndrome in university students. Coll Stud J. 2001;35(3):472-6.

10. Hicks RA, Fernandez C, Pellegrini RJ. The changing sleep habits of university students: an update. Percept Mot Skills. 2001;93(3).

11. Taylor DJ, Bramoweth AD. Patterns and consequences of inadequate sleep in college students: substance use and motor vehicle accidents. J Adolesc Health. 2010;46(6):610-2.

12. Ruiter ME, Decoster J, Jacobs L, Lichstein KL. Normal sleep in African-Americans and Caucasian-Americans: a meta-analysis. Sleep Med. 2011;12(3):209-14.

13. Edens KM. The relationship of university students' sleep habits and academic motivation. NASPA J. 2006;43(3):432-45.

14. Taylor DJ, Bramoweth AD, Grieser EA, Tatum JI, Roane BM. Epidemiology of insomnia in college students: relationship with mental health, quality of life, and substance use difficulties. Behav Ther. 2013;44(3):339-48. doi:10.1016/j.beth.2012.12.001.

15. Kyle SD, Morgan K, Espie CA. Insomnia and health-related quality of life. Sleep Med Rev. 2010;14(1):69-82. doi:10.1016/j.smrv.2009. 07.004.

16. Forquer LM, Camden AE, Gabriau KM, Johnson CM. Sleep patterns of college students at a public university. J Am Coll Heal. 2008;56(5): $563-5$.

17. Carney CE, Edinger JD, Meyer BR, Lindman L, Istre T. Daily activities and sleep quality in college students. Chronobiol Int. 2006;23(3):623-37. doi:10.1080/07420520600650695.

18. Clegg-Kraynok MM, McBean AL, Montgomery-Downs HE. Sleep quality and characteristics of college students who use prescription psychostimulants nonmedically. Sleep Med. 2011;12(6):598-602. doi:10.1016/j.sleep.2011.01.012.

19. Gilbert SP, Weaver CC. Sleep quality and academic performance in university students: a wake-up call for college psychologists. J Coll Stud Psychother. 2010;24(4):295-306.

20. Mesquita G, Reimao R. Quality of sleep among university students: effects of nighttime computer television use. Arq Neuropsiquiatr. 2010;68(5):720-5.

21. Foley D, Ancoli-Israel S, Britz P, Walsh J. Sleep disturbances and chronic disease in older adults: results of the 2003 National Sleep Foundation Sleep in America Survey. J Psychosom Res. 2004;56(5): 497-502.

22. Petersen H, Kecklund G, D’Onofrio P, Nilsson J, Åkerstedt T. Stress vulnerability and the effects of moderate daily stress on sleep polysomnography and subjective sleepiness. J Sleep Res. 2013;22(1):50-7. doi:10.1111/j.1365-2869.2012.01034.x.

23. Stein M, Mendelsohn J, Obermeyer W, Amromin J, Benca R. Sleep and behavior problems in school-aged children. Pediatrics. 2001;107(4):1-9.

24. Turner R, Drummond S, Salamat J, Brown G. Effects of $42 \mathrm{hr}$ of total sleep deprivation on component processes of verbal working memory. Neuropsychology. 2007;21(6):787-95.

25. Voinescu BI, Szentagotai A, David D. Sleep disturbance, circadian preference and symptoms of adult attention deficit hyperactivity disorder (ADHD). J Neural Transm. 2012;119(10):1195-204. doi: 10.1007/s00702-012-0862-3. 
26. Yen C-F, King BH, Tang T-C. The association between short and long nocturnal sleep durations and risky behaviours and the moderating factors in Taiwanese adolescents. Psychiatry Res. 2010;179(1): 69-74. doi:10.1016/j.psychres.2009.02.016.

27. Komada Y, Inoue Y, Hayashida K, Nakajima T, Honda M, Takahashi $\mathrm{K}$. Clinical significance and correlates of behaviorally induced insufficient sleep syndrome. Sleep Med. 2008;9(8):851-6. doi:10.1016/j. sleep.2007.08.018.

28. Gaultney JF. The prevalence of sleep disorders in college students: impact on academic performance. J Am Coll Heal. 2010;59(2):91-7.

29. Kloss JD, Nash CO, Horsey SE, Taylor DJ. The delivery of behavioral sleep medicine to college students. J Adolesc Health. 2011;48(6):553-61. doi:10.1016/j.jadohealth.2010.09.023.

30. Obermeyer WH, Benca RM. Effects of drugs on sleep. Neurol Clin. 1996;14(4):827-40.

31. Adam E, Snell E, Pendry P. Sleep timing and quantity in ecological and family context: a nationally representative time-diary study. J Fam Psychol. 2007;21(1):4-19.

32. Gillen-O’Neel C, Huynh VW, Fuligni AJ. To study or to sleep? The academic costs of extra studying at the expense of sleep. Child Dev. 2013;84(1):133-42.

33. Carskadon MA, Wolfson AR, Acebo C, Tzischinsky O, Seifer R. Adolescent sleep patterns, circadian timing, and sleepiness at a transition to early school days. Sleep J Sleep Res Sleep Med. 1998;21(8):871-81.

34. Jenaro C, Flores N, Gomez-Vela M, Gonzalez-Gil F, Caballo C. Problematic Internet and cell-phone use: psychological behavioral, and health correlates. Addict Res Theory. 2007;15(3):309-20.

35. Johns M. A new method for measuring daytime sleepiness: the Epworth Sleepiness Scale. Sleep. 1991;14(6):540-5.
36. Buysse DJ, Hall ML, Strollo PJ, Kamarck TW, Owens J, Lee L, et al. Relationships between the Pittsburgh Sleep Quality Index (PSQI), Epworth Sleepiness Scale (ESS), and clinical/polysomnographic measures in a community sample. J Clin Sleep Med. 2008;4(6): $563-71$.

37. Gibson E, Powles A, Thabane L, O'Brien S, Molnar D, Trajanovic N, et al. "Sleepiness" is serious in adolescence: two surveys of 3235 Canadian students. BMC Public Health. 2006;6:1-9.

38. Buysse DJ, Reynolds 3rd CF, Monk TH, Berman SR, Kupfer DJ. The Pittsburgh Sleep Quality Index: a new instrument for psychiatric practice and research. Psychiatry Res. 1989;28(2): 193-213.

39. Carpenter JS, Andrykowski MA. Psychometric evaluation of the Pittsburgh Sleep Quality Index. J Psychosom Res. 1998;45(1):513. doi:10.1016/S0022-3999(97)00298-5.

40. Streiner DL. Starting at the beginning: an introduction to coefficient alpha and internal consistency. J Pers Assess. 2003;80(1):99-103.

41. Fay MP, Proschan MA. Wilcoxon-Mann-Whitney or t-test? On assumptions for hypothesis tests and multiple interpretations of decision rules. Stat Surv. 2010;4:1-39.

42. Medeiros ALD, Mendes DBF, Lima PF, Araujo JF. The relationships between sleep-wake cycle and academic performance in medical students. Biol Rhythm Res. 2001;32(2):26370.

43. Crowley SJ, Acebo C, Carskadon MA. Sleep, circadian rhythms, and delayed phase in adolescence. Sleep Med. 2007;8(6):602-12.

44. Nakamura J, Csikszentmihalyi M, Snyder SJLCR. Flow theory and research. Oxford handbook of positive psychology (2nd edn). Oxford library of psychology. New York: Oxford University Press; 2009. p. 195-206. 